\title{
Alternativas na fertilização de feijão visando a reduzir a aplicação de $\mathrm{N}$-ureia ${ }^{1}$
}

\author{
Juliano Garcia Bertoldo ${ }^{2}$, Amanda Pelisser ${ }^{2}$, \\ Raquel Paz da Silva ${ }^{2}$, Rodrigo Favreto ${ }^{2}$, Luciene Antunes Dias de Oliveira ${ }^{2}$
}

\begin{abstract}
Alternatives in bean fertilization

to reduce the application of $\mathrm{N}$-urea

As a result of the increasing use of chemical nitrogen fertilizers and their economic and environmental impacts, it is necessary to search for alternative methods to improve soil fertility, such as biological nitrogen fixation and/or alternative supplements of this nutrient. This study aimed at evaluating the effects of inoculation and application of seaweed extract, molybdenum and rock powder, as an alternative to the application of urea, in beans. A randomized blocks design, with three replications, was used. Two common bean cultivars (Pérola and FEPAGRO 26), as well as the following cultivation methods, were used: $i$ ) conventional; ii) inoculation with a mixture of three inoculants recommended for bean; iii) inoculation + complementation via seeds, with extracts of the Ascophyllum nodosum seaweed and mineral fertilizer containing rock powder and molybdenum; iv) inoculation + complementation via leaf, using products with extracts of the Ascophyllum nodosum seaweed and mineral fertilizer containing rock powder and molybdenum. The inoculation alone is not enough to reach grain yield levels similar to those reached using the conventional method. The cultivation method with inoculation + complementation via seeds maintains grain yield with the lowest cost, in relation to the conventional method.
\end{abstract}

KEY-WORDS: Phaselous vulgaris L.; nitrogen fertilizers; seaweed extract; molybdenum; rock powder.

\section{INTRODUÇÃO}

A produtividade de feijão, no Brasil, pode ser considerada extremamente baixa (cerca de $900 \mathrm{~kg} \mathrm{ha}^{-1}$, Conab 2014), não refletindo o potencial de rendimento das atuais cultivares recomendadas $\left(>2.000 \mathrm{~kg} \mathrm{ha}^{-1}\right)$. Dentre os fatores que podem estar contribuindo para essa realidade estão o cultivo em locais de baixa fertilidade dos solos; o baixo uso de insumos (Barbosa Filho et al. 2005), resultando em inadequada nutrição mineral da cultura (Binotti et

\section{RESUMO}

Em virtude da crescente utilização de fertilizantes nitrogenados químicos, e seus impactos econômico e ambiental, é necessário buscar métodos alternativos para melhorar a fertilidade do solo, como, por exemplo, a fixação biológica de nitrogênio e/ou suplementos alternativos desse nutriente. Objetivou-se avaliar os efeitos da inoculação e aplicação de extrato de alga, molibdênio e pó de rocha, como alternativa à aplicação de ureia, em feijão. O delineamento experimental foi o de blocos ao acaso, com três repetições. Foram utilizadas duas cultivares de feijão (Pérola e FEPAGRO 26), bem como os seguintes métodos de cultivo: $i$ ) convencional; $i i$ ) inoculação com a mistura de três inoculantes recomendados para o feijão; iii) inoculação + complementação via sementes, com extrato da alga Ascophyllum nodosum e fertilizante mineral contendo pó de rocha e molibdênio; $i v$ ) inoculação + complementação via foliar, utilizando-se produtos com extrato da alga Ascophyllum nodosum e fertilizante mineral contendo pó de rocha e molibdênio. A inoculação, por si só, não é suficiente para atingir patamares similares aos do método convencional, no rendimento de grãos. O método de cultivo inoculação + complementação via sementes propicia a manutenção do rendimento de grãos com menor custo, em relação ao método de cultivo convencional.

PALAVRAS-CHAVES: Phaselous vulgaris L.; fertilizantes nitrogenados; extrato de alga; molibdênio; pó de rocha.

al. 2009, Biscaro et al. 2011); e, provavelmente, a incipiência no uso da fixação biológica de nitrogênio (FBN), por meio da técnica de inoculação.

Entre 1960 e 2000, o consumo global de fertilizantes aumentou mais de dez vezes: de $10,8 \mathrm{Mg}$ (toneladas) para 113 milhões de $\mathrm{Mg}$, por ano (Crews \& Peoples 2004). Práticas agrícolas intensivas, que proporcionam maiores rendimentos e qualidade, requerem uso extensivo de fertilizantes e pesticidas químicos, que podem elevar o custo de produção e causar danos ambientais (Tozlu et al. 2012). 
Apesar do aumento na produtividade resultante do uso intensivo desses insumos, essa prática requer alta energia e gera custos ambientais. Sendo assim, torna-se necessária a busca por métodos alternativos para melhoria da fertilidade do solo e manejo de insetos e doenças (Gopalakrishnan et al. 2014).

Os solos de regiões tropicais, geralmente, são deficientes em nitrogênio $(\mathrm{N})$, devido aos baixos teores de matéria orgânica, sendo considerado esse um dos fatores limitantes à produção agrícola (Souza \& Moreira 2011, Silva et al. 2012). Em contrapartida, fontes alternativas de nitrogênio estão disponíveis, como, por exemplo, a FBN.

A associação do feijão com espécies de bactérias do grupo dos rizóbios, principalmente Rhizobium tropici, é uma tecnologia capaz de substituir a adubação nitrogenada, total ou parcialmente, reduzindo o custo de produção e proporcionando altos rendimentos na cultura (Grange et al. 2007, Pelegrin et al. 2009, Tozlu et al. 2012). No entanto, a disponibilização de tecnologias alternativas para melhorar a produção agrícola, de forma sustentável, está aquém do desejado (Souza \& Moreira 2011). Com exceção do seu sucesso na cultura da soja, no Brasil (Alves et al. 2003), onde o melhoramento genético para a FBN iniciou-se em 1930 (Alcântara et al. 2009), o processo de FBN é pouco explorado nos sistemas agrícolas modernos.

Nesses sistemas, os ciclos de cultivo cada vez mais curtos não são suficientes para que o aparato simbiótico fixador de $\mathrm{N}$ se estabeleça e funcione de maneira eficiente. Consequentemente, os agricultores utilizam fertilizantes nitrogenados produzidos sinteticamente, principalmente ureia, para suprir as necessidades imediatas de plantas e animais por esse nutriente (Neves et al. 2002). Ademais, ao contrário do que ocorre com a soja, nos cultivos atuais de feijão, a FBN não é capaz de suprir a necessidade de $\mathrm{N}$ da cultura (Matoso \& Kusdra 2014).

Tendo-se em vista que a técnica de inoculação com rizóbios, por si só, ainda não garante o fornecimento de $\mathrm{N}$ ao feijão, é necessário estudar alternativas para aumentar a eficiência da FBN, incrementando a produtividade e reduzindo o impacto ambiental da cultura. Além de uma adubação adequada e eficiente uso da FBN, outras técnicas que possam beneficiar principalmente os pequenos agricultores de feijão são bem-vindas, sobretudo aquelas em que o custo e os possíveis impactos ambientais sejam reduzidos. A aplicação de pó de rocha, molibdênio e extrato de alga na lavoura, como complementação à FBN, são alternativas com esses requisitos.

Estudos evidenciaram que a aplicação de pó de rocha, molibdênio e extrato de alga pode ser benéfica ao feijão, gerando incremento à $\mathrm{FBN}$, aumento na produtividade, tolerância a estresses de ambiente e aumento no teor de clorofila e da capacidade fotossintética, dentre outros (Theodoro \& Leonardos 2006, Ferreira et al. 2009, Matysiak et al. 2011, Vasconcelos \& Gonçalvez 2013, Matoso \& Kusdra 2014).

Os extratos de algas marinhas, como a Ascophyllum nodosum, possuem diferentes reguladores de crescimento (citocininas, auxinas, giberelinas e betaínas), macronutrientes ( $\mathrm{Ca}, \mathrm{K}$ e $\mathrm{P}$ ) e micronutrientes ( $\mathrm{Fe}, \mathrm{Cu}, \mathrm{Zn}, \mathrm{B}, \mathrm{Mn}$, Co e $\mathrm{Mo}$ ) que podem beneficiar as plantas (Durand et al. 2003, Khan et al. 2009 ), além de serem inócuos.

O molibdênio é considerado elemento essencial para as plantas, por ser componente das enzimas nitrato redutase e nitrogenase (Lopes et al. 2014). Existem evidências de que o molibdênio gera incrementos na produtividade e na nodulação do feijão e de outras culturas (Rocha et al. 2011, Rossi et al. 2012, Lopes et al. 2014).

O uso do pó de rocha apresenta diversas vantagens, em relação aos fertilizantes solúveis. Entre essas, cita-se o fato de ser considerado ecologicamente correto, apresentar custo reduzido, propiciar maior velocidade de crescimento e sanidade das plantas, além de melhorar a fertilidade do solo e, possivelmente, o incremento na produtividade (Santos et al. 2001, Ferreira et al. 2009, Plewka et al. 2009, Camargo et al. 2012).

Assim, objetivou-se, com este estudo, avaliar os efeitos da inoculação de bactérias fixadoras de nitrogênio, juntamente com a aplicação de pó de rocha, molibdênio e extrato de alga, como alternativa à aplicação de ureia, em feijão.

\section{MATERIAL E MÉTODOS}

O estudo foi conduzido em área experimental da Fundação Estadual de Pesquisa Agropecuária (Fepagro), em Maquiné (RS) (2954'S, 50 $19^{\prime} \mathrm{W}$ e altitude de $38 \mathrm{~m})$, cujo clima é do tipo Cfa, com precipitação anual de $1.679,3 \mathrm{~mm}$ (Matzenauer et al. 2011). O solo da área experimental é do tipo Chernossolo Háplico órtico típico (Unidade Vila) (Anama/UFRGS 2000). O experimento foi instalado 
em dois períodos: na safra e safrinha de 2013/2014, com semeaduras em outubro de 2013 e fevereiro de 2014, respectivamente.

O delineamento experimental foi o de blocos ao acaso, com três repetições. Cada parcela foi constituída de quatro linhas de 4,0 $\mathrm{m}$ de comprimento, espaçadas em $0,45 \mathrm{~m}$, perfazendo $7,2 \mathrm{~m}^{2}$ de área total. A área útil foi composta pelas duas linhas centrais, totalizando $3,6 \mathrm{~m}^{2}$, e a densidade de semeadura foi de 12 plantas $\mathrm{m}^{-1}$. Por ocasião da semeadura, todos os tratamentos receberam $275 \mathrm{~kg} \mathrm{ha}^{-1}$ de fertilizante mineral NPK de fórmula 5-20-20. O controle de plantas daninhas foi realizado com uma aplicação de Fluazifop-p-butyl + fomesafen, na dose de $1 \mathrm{~L} \mathrm{ha}^{-1}$, e o de pragas com uma aplicação de Metamidophos (1 Lha $\left.{ }^{-1}\right)$, de acordo com as necessidades da cultura.

Os tratamentos foram distribuídos no esquema fatorial $2 \times 2 \times 4$, sendo constituídos de duas cultivares comerciais (Pérola - carioca; FEPAGRO 26 - preto), duas épocas de cultivo (safra e safrinha) e quatro métodos de cultivo de feijão: $i$ ) convencional (CONV): aplicação de N (45\% de $\mathrm{N}$ na dose de $70 \mathrm{~kg} \mathrm{ha}^{-1}$, na cobertura, 30 dias após a emergência, no estádio V4); ii) inoculante (INOC): inoculação das sementes com a mistura de três inoculantes recomendados para o feijão (SEMIA 4077, SEMIA 4080 e SEMIA 4088, na dose de $200 \mathrm{~g}$ de inoculante para $50 \mathrm{~kg}$ de sementes); iii) inoculante + complementação via sementes (INOC + CS): inoculação das sementes com a mistura dos mesmos três inoculantes recomendados para o feijão (tratamento $i i$ ) e tratamento de sementes com produtos com extrato da alga Ascophyllum nodosum (Acadian ${ }^{\circledR}$ ), fertilizante mineral contendo pó de rocha $\left(\right.$ Gigamix $\left.^{\circledR}\right)$ e molibdênio $\left(\right.$ AgrComo $\left.^{\circledR}\right)$, de acordo com a recomendação do fabricante

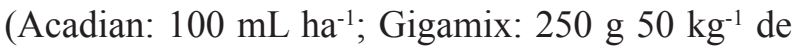
sementes; AgrComo: $\left.100 \mathrm{~mL} \mathrm{ha}^{-1}\right)$; iv) inoculante + complementação via foliar (INOC $+\mathrm{CF}$ ): inoculação das sementes com a mistura dos mesmos três inoculantes recomendados para o feijão (tratamento $i$ ) e três aplicações via foliar da mistura dos produtos com extrato da alga Ascophyllum nodosum (Acadian ${ }^{\circledR}$ ), fertilizante mineral contendo pó de rocha $\left(\mathrm{Gigamix}^{\circledR}\right)$ e molibdênio $\left(\mathrm{AgrComo}^{\circledR}\right)$, nos estádios V4, R6 e $\mathrm{R} 8$, de acordo com a recomendação do fabricante

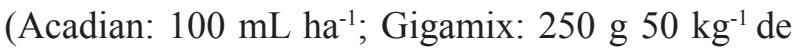
sementes; AgrComo: $100 \mathrm{~mL} \mathrm{ha}^{-1}$ ).

Durante o desenvolvimento da cultura, foram realizadas avaliações morfoagronômicas, seguindo-se descritores mínimos específicos da cul- tura (Schoonhoven \& Pastor-Corrales 1987). A quantificação dos índices de clorofila (CLO) existentes nas folhas foi realizada em pleno florescimento, com três leituras por planta, em cinco plantas por parcela, totalizando 15 leituras. Esse índice CLO foi quantificado com o auxílio de clorofilômetro (ClorofiLOG ${ }^{\circledR}$ modelo CFL 1030, produzido pela Falker Automação Agrícola). Foram avaliados, também, os caracteres emergência de plantas (EME), índice de clorofila (CLOa, CLOb e CLOt), número de nódulos totais (NNT), massa seca aérea (MSA, em g), massa seca da raiz (MSR, em g), estatura de planta (EST, em $\mathrm{cm}$ ), diâmetro do caule (DIC, em $\mathrm{cm}$ ), número de legumes por planta (NLP), número de grãos por legume (NGL), peso de cem sementes (PCS, em g), rendimento de grãos (REND, em kg ha ${ }^{-1}$ ) e rendimento biológico ou biomassa total (RBIO, em g).

As avaliações dos quatro sistemas de cultivo foram realizadas no estádio de florescimento (R6) e maturação de colheita (R9). Os dados foram submetidos à análise de variância conjunta, pelo teste $\mathrm{F}$, a $5 \%$, e posterior teste de comparação de médias, pelo teste Tukey $(\mathrm{p}<0,05)$. O pacote estatístico utilizado foi o SAS University Edition ${ }^{\circledR}$ (SAS INSTITUTE 2014).

Para os cálculos da eficiência econômica de cada estratégia de complementação, foram considerados os seguintes parâmetros: a) para o cálculo da hora-máquina $(\mathrm{hm})$, foi utilizada a planilha da Coordenadoria de Assistência Técnica Integral do Instituto de Economia Agrícola do Estado de São Paulo (IEA 2014). A planilha inclui custos fixos e variáveis do trator e dos implementos, inclusive a mão de obra do operador. O valor aproximado para 1 $\mathrm{hm}$ foi de $\mathrm{R} \$ 65,00 ; \mathrm{b})$ a estimativa da hora-homem (hh) foi obtida a partir de pesquisas próprias realizadas no município de Maquiné, onde um safrista ganha, em média, $\mathrm{R} \$ 65,00$ (líquido), por dia, representando custo de mão de obra para o produtor de $\mathrm{R} \$ 72,15$ (com a inclusão dos $11 \%$ de INSS); c) para cada pulverização do extrato de alga, pó de rocha e molibdênio, são necessárias 2 horas-máquina $(\mathrm{hm})$, 1,5 hm para a adubação de cobertura mecanizada (aplicação de ureia) e, no tratamento manual de sementes, são necessárias 0,25 hh (Wander \& Abreu 2005); d) considerou-se que o trabalho foi realizado por um safrista, com o auxílio de um trator $75 \mathrm{cv}$, um distribuidor rotativo de $400 \mathrm{~L}$ e um pulverizador de 400 L, 10 m e 20 bicos; e) os preços dos produtos, insumos e serviços utilizados foram obtidos por meio de pesquisa no comércio local, em julho de 2014. 


\section{RESULTADOS E DISCUSSÃO}

$\mathrm{Na}$ análise de variância conjunta, foram verificadas diferenças significativas entre as cultivares para a maioria dos caracteres analisados (Tabela 1). No entanto, não foi observada interação dos fatores avaliados para nenhum caractere, demonstrando que as cultivares apresentaram comportamento similar, inerente aos métodos utilizados. Esse resultado permite inferir que é possível ampliar a utilização das metodologias empregadas, independentemente da cultivar.

A cultivar Pérola (carioca) apresentou superioridade nos caracteres emergência de plantas, índice de clorofila, massa seca da raiz, estatura, peso de cem sementes e rendimento biológico, e a FEPAGRO 26 (preto) foi superior nos caracteres diâmetro de caule, número de legumes por planta e número de grãos por legume. Para os caracteres massa seca aérea e rendimento de grãos, não houve diferenças entre as cultivares. Pode ser observado que a cultivar Pérola apresentou superioridade na maior parte dos caracteres iniciais do desenvolvimento (estádio R6). Porém, ao final do ciclo, as duas cultivares se equivaleram, sendo a FEPAGRO 26, inclusive, superior em alguns caracteres. Esse resultado confirma as avaliações a campo de que a FEPAGRO 26 tem crescimento inicial lento, porém, a partir do estádio R6, ocorre incremento substancial no desenvolvimento, evidenciando todo o seu potencial produtivo.

Os métodos de cultivo tiveram influência significativa para alguns dos caracteres (Tabela 2). Nos caracteres iniciais do desenvolvimento (até o estádio R6), não houve diferenças significativas entre os métodos de cultivo utilizados, com exceção dos caracteres emergência de plantas e número de nódulos totais, para os quais o método convencional (CONV) foi inferior aos demais (Tabela 2). No método CONV, ou seja, o habitualmente utilizado pelos produtores, para o feijão, verificou-se menor quantidade de nódulos por planta e menor estande inicial de plantas, quando comparado aos métodos de cultivo com inoculação (INOC) e/ou complementação via sementes ou foliar (INOC + CS e INOC + $\mathrm{CF}$, respectivamente). A aplicação de ureia pode ter inibido a formação de nódulos, e efeito oposto pode ter ocorrido com a inoculação.

Por outro lado, a utilização dos produtos complementares à inoculação (extrato de alga, molibdênio e pó de rocha) não influenciou no incremento desses caracteres. No entanto, apesar de não haver diferenças significativas, pode ser observado que as médias, no método de cultivo INOC $+\mathrm{CS}$, foram próximas ou maiores do que no método $\mathrm{CONV}$, em todos os caracteres, até o estádio R6 (Tabela 2).

Na maturação da colheita (estádio R9), verificou-se que os métodos de cultivo não apresentaram diferenças significativas para os caracteres estatura de planta, número de grãos por legume e rendimento biológico (Tabela 2). O método de cultivo CONV foi igual aos demais métodos para os caracteres diâmetro do caule, número de legumes por planta, peso de cem sementes e rendimento de grãos, com exceção do método INOC, que foi inferior para esses caracteres.

Esses resultados indicam que a técnica de inoculação atual, por si só, não é suficiente para atingir patamares de produção ou melhoria no desenvolvimento da planta de feijão, sendo necessária

Tabela 1. Comparação de médias de duas cultivares de feijão, no estádio de florescimento (R6) e maturação de colheita (R8), na análise conjunta da época da safra e da safrinha (Maquiné, RS, 2013/2014).

\begin{tabular}{|c|c|c|c|c|c|c|c|}
\hline \multirow{2}{*}{ Cultivares } & \multicolumn{7}{|c|}{ Caracteres em R6* } \\
\hline & EME & CLOa & $\mathrm{CLOb}$ & CLOt & NNT & MSA & MSR \\
\hline Pérola & $213,29 \mathrm{a}$ & $35,94 \mathrm{a}$ & $9,87 \mathrm{a}$ & $45,81 \mathrm{a}$ & $27,18 \mathrm{a}$ & $7,74 \mathrm{a}$ & $0,88 \mathrm{a}$ \\
\hline FEPAGRO 26 & $184,29 \mathrm{~b}$ & $34,40 \mathrm{~b}$ & $8,66 \mathrm{~b}$ & $43,06 \mathrm{~b}$ & $25,78 \mathrm{a}$ & $7,53 \mathrm{a}$ & $0,75 \mathrm{~b}$ \\
\hline Média & 198,79 & 35,17 & 9,26 & 44,44 & 26,47 & 7,64 & 0,81 \\
\hline \multirow{2}{*}{ Cultivares } & \multicolumn{7}{|c|}{ Caracteres em R8* } \\
\hline & EST & DIC & NLP & NGL & PCS & REND & RBIO \\
\hline Pérola & $77,52 \mathrm{a}$ & $0,45 \mathrm{~b}$ & $7,93 \mathrm{~b}$ & $4,12 b$ & $21,28 \mathrm{a}$ & $1.116 \mathrm{a}$ & $1.211 \mathrm{a}$ \\
\hline FEPAGRO 26 & $46,91 \mathrm{~b}$ & $0,53 \mathrm{a}$ & $9,91 \mathrm{a}$ & $5,37 \mathrm{a}$ & $18,09 \mathrm{~b}$ & $1.107 \mathrm{a}$ & $996 \mathrm{~b}$ \\
\hline Média & 62,21 & 0,49 & 8,90 & 4,75 & 19,69 & 1.111 & 1.105 \\
\hline
\end{tabular}


a sua complementação. Neste trabalho, optou-se pela complementação com produtos "ecologicamente" mais sustentáveis e de menor custo ao agricultor.

Ao contrário da soja, no feijão, a FBN, isoladamente, não é capaz de suprir a necessidade de $\mathrm{N}$ da cultura (Matoso \& Kusdra 2014). Nesse caso, a inoculação das sementes com a utilização dos produtos em associação (pó de rocha, molibdênio e extrato de alga) pode propiciar efeito benéfico para a cultura, uma vez que reduz a quantidade de ureia aplicada ao cultivo e aumenta a produtividade, em relação ao uso da inoculação isoladamente.

Um detalhe importante é que, com a inoculação das sementes (exceto no método CONV), o número de nódulos foi maior. Aliado ao incremento no número de nódulos, o método de cultivo INOC + CS propiciou rendimento de grãos equivalente ao método CONV, assim como o método INOC + CF. Porém, é importante ressaltar que a complementação foliar $(\mathrm{INOC}+\mathrm{CF})$ é mais onerosa do que a efetuada via sementes (INOC + CS) (Tabela 3). Assim, a utilização do método de cultivo INOC + CS pode ser uma alternativa, tanto do ponto de vista econômico quanto ambiental, pois reduz, consideravelmente, o uso de ureia na lavoura e permite a manutenção do rendimento de grãos.

A ureia é obtida por meio de processo que utiliza o nafta, um derivado do petróleo. Assim,

Tabela 2. Efeito de quatro métodos de cultivo [convencional (CONV), inoculação (INOC), inoculação com complementação via sementes (INOC + CS) e inoculação com complementação via foliar (INOC + CF)], avaliados em feijão, no estádio de florescimento (R6) e maturação de colheita (R9), na análise conjunta da época da safra e da safrinha (Maquiné, RS, 2013/2014).

\begin{tabular}{|c|c|c|c|c|c|c|c|}
\hline \multirow{2}{*}{ Métodos } & \multicolumn{7}{|c|}{ Caracteres em R6* } \\
\hline & EME & $\mathrm{CLOa}$ & $\mathrm{CLOb}$ & CLOt & NNT & MSA & MSR \\
\hline CONV & $189,92 \mathrm{~b}$ & $36,03 \mathrm{a}$ & $9,55 \mathrm{a}$ & $45,58 \mathrm{a}$ & $18,90 \mathrm{~b}$ & $7,71 \mathrm{a}$ & $0,79 \mathrm{a}$ \\
\hline INOC & $201,50 \mathrm{a}$ & $33,85 \mathrm{a}$ & $8,67 \mathrm{a}$ & $42,52 \mathrm{a}$ & $30,73 \mathrm{a}$ & $6,94 \mathrm{a}$ & $0,78 \mathrm{a}$ \\
\hline $\mathrm{INOC}+\mathrm{CS}$ & $203,75 \mathrm{a}$ & $35,56 \mathrm{a}$ & $9,62 \mathrm{a}$ & $45,18 \mathrm{a}$ & $29,42 \mathrm{a}$ & $7,66 \mathrm{a}$ & $0,85 \mathrm{a}$ \\
\hline $\mathrm{INOC}+\mathrm{CF}$ & $200,00 \mathrm{a}$ & $35,25 \mathrm{a}$ & $9,22 \mathrm{a}$ & $44,47 \mathrm{a}$ & $26,87 \mathrm{a}$ & $8,27 \mathrm{a}$ & $0,83 \mathrm{a}$ \\
\hline Média & 198,79 & 35,17 & 9,26 & 44,44 & 26,47 & 7,64 & 0,81 \\
\hline \multirow{2}{*}{ Métodos } & \multicolumn{7}{|c|}{ Caracteres em R9* } \\
\hline & EST & DIC & NLP & NGL & PCS & REND & RBIO \\
\hline CONV & $63,67 \mathrm{a}$ & $0,51 \mathrm{a}$ & $9,03 \mathrm{a}$ & 4,96 a & $19,56 \mathrm{ab}$ & $1.283 \mathrm{a}$ & $1.136 \mathrm{a}$ \\
\hline INOC & $58,79 \mathrm{a}$ & $0,44 \mathrm{~b}$ & $7,69 \mathrm{~b}$ & $4,92 \mathrm{a}$ & $18,80 \mathrm{~b}$ & $887 \mathrm{~b}$ & $1.021 \mathrm{a}$ \\
\hline $\mathrm{INOC}+\mathrm{CS}$ & $60,78 \mathrm{a}$ & $0,49 \mathrm{ab}$ & $8,70 \mathrm{ab}$ & $4,55 \mathrm{a}$ & $20,78 \mathrm{a}$ & $1.185 \mathrm{a}$ & $1.164 \mathrm{a}$ \\
\hline $\mathrm{INOC}+\mathrm{CF}$ & $65,53 \mathrm{a}$ & $0,51 \mathrm{a}$ & $10,27 \mathrm{a}$ & $4,58 \mathrm{a}$ & $19,61 \mathrm{ab}$ & $1.099 \mathrm{a}$ & $1.095 \mathrm{a}$ \\
\hline Média & 62,21 & 0,49 & 8,90 & 4,75 & 19,69 & 1.111 & 1.105 \\
\hline
\end{tabular}

Tabela 3. Eficiência econômica entre quatro métodos de cultivo [convencional (CONV), inoculação (INOC), inoculação com complementação via sementes (INOC + CS) e inoculação com complementação via foliar (INOC + CF)], em feijão, na análise conjunta da época da safra e da safrinha (Maquiné, RS, 2013/2014).

\begin{tabular}{lcrrrrr}
\hline \multirow{2}{*}{ Métodos } & PROD $^{1}$ & \multicolumn{1}{c}{ RB $^{2}$} & $\mathrm{II}^{3}$ & $\mathrm{RL}^{4} \mathrm{C}^{4}$ & $\mathrm{RG}^{5}$ & $\mathrm{PR}^{6}$ \\
\cline { 2 - 6 } & sacas ha $^{-1}$ & & $\mathrm{R} \$$ & & \multicolumn{1}{c}{ dias } \\
\hline CONV & 20,64 & $2.064,45$ & 194,94 & $1.869,51$ & 9,44 & 33 \\
INOC & 14,78 & $1.478,00$ & 14,38 & $1.463,62$ & 0,97 & 4 \\
INOC + CS & 19,75 & $1.975,32$ & 40,63 & $1.934,69$ & 2,06 & 8 \\
INOC + CF & 18,32 & $1.831,90$ & 430,63 & $1.401,27$ & 23,51 & 64 \\
\hline
\end{tabular}

Produtividade (PROD): quantidade de sacas de $60 \mathrm{~kg}$ colhidas por hectare; ${ }^{2}$ Receita bruta (RB): quantidade de sacas de $60 \mathrm{~kg}$ colhidas, multiplicada pelo preço médio praticado no período (R\$ 100,00 a saca); ${ }^{3}$ Investimento isolado (II): somatório dos dispêndios que diferenciam cada tratamento, quais sejam: aplicação de ureia e molibdênio foliar, tratamento de sementes e mão de obra para a realização desses serviços (foram desconsiderados todos os demais custos comuns a todos os tratamentos); ${ }^{4}$ Receita líquida para comparação (RL-C): receita bruta menos o investimento isolado; ${ }^{5}$ Receita gasta (RG): porcentagem da receita bruta gasta com o investimento isolado; ${ }^{6}$ Prazo de retorno do investimento isolado (PR, em dias): dias necessários para recuperar o investimento isolado realizado, considerando-se como zero todos os demais custos da lavoura que são comuns a todos os tratamentos II, em relação à RL-C. 
não consegue atender os princípios de conservação dos recursos não renováveis e de minimização da poluição (Neves et al. 2002). A fixação de $\mathrm{N}_{2}$ tem papel fundamental na manutenção da produção agrícola mundial (Herridge \& Rose 2000) e gera menor impacto ambiental. A incorporação de nitrogênio via fixação biológica é, naturalmente, bastante significativa, nos diferentes ecossistemas do planeta, representando uma importante economia de energia fóssil (Alcântara et al. 2009) e podendo reduzir os custos de produção (Hungria \& Vargas 2000).

Além dos benefícios supracitados, a inoculação pode ser fundamental em sistema plantio direto, que é recomendado na agricultura tropical sempre que possível (CTSBF 2010). Para a obtenção de altas produtividades, a curto e longo prazo, é importante o emprego de tecnologias sustentáveis, como o plantio direto (Gerlach et al. 2013). Entretanto, nesse sistema, pode ser necessário utilizar maiores quantidades de $\mathrm{N}$, em função do processo de imobilização dos nutrientes, limitando a produtividade da cultura (Lemos et al. 2008). Assim, o agricultor, provavelmente, tem um ônus econômico, com uma maior necessidade de fertilizantes nitrogenados. O método de cultivo INOC $+\mathrm{CS}$ pode ser uma boa alternativa para o plantio direto, pois depende de menor quantidade de ureia, tendo como principal fonte de $\mathrm{N}$ a FBN, reduzindo os custos.

Apesar da evidente melhoria, do ponto de vista agronômico e ambiental, outro aspecto relevante é o custo de produção desses métodos alternativos de fertilização nitrogenada. A fim de obter uma medida econômica de comparação entre os tratamentos, foram calculados a receita bruta, o investimento isolado, a receita líquida para comparação, o percentual da receita bruta gasta e o prazo de retorno do investimento isolado (Tabela 3).

Os resultados demonstram que o maior retorno por Real investido foi obtido com o método INOC, pois, mesmo tendo a menor receita bruta (a única com REND estatisticamente diferente), seu custo foi o mais baixo de todos os métodos. Assim, a inoculação sem complementação de $\mathrm{N}$ pode ser recomendada para os agricultores que tiverem poucos recursos para investimento.

Já com o método INOC + CS, foi obtida a maior renda líquida, sendo recomendado para a maioria dos agricultores. Mesmo não sendo o mais baixo, o custo do método INOC + CS está próximo ao do INOC, e o seu rendimento é estatisticamente igual ao do método CONV.
Ambos os métodos recomendados aplicam-se aos produtos diretamente na semente, antes da semeadura, necessitando-se de menos horas-homem e horas-máquina que os demais métodos de cultivo (que utilizam ureia em cobertura ou via pulverização foliar). Com isso, esses métodos são consideravelmente mais simples, mais seguros e resultam em menor impacto ambiental. No conjunto de indicadores, o método INOC + CS pode ser considerado o mais promissor, em relação ao CONV, pois, além de manter o mesmo patamar de rendimento, gera retorno financeiro maior.

Esses resultados são de grande relevância para novas alternativas no cultivo de feijão, principalmente na complementação à prática da inoculação, dado que as atuais cultivares apresentam baixa capacidade de FBN.

O feijão é altamente dependente de ureia, apesar de ser uma leguminosa e passível de haver FBN. Essa necessidade difere da soja, na qual não são utilizados fertilizantes nitrogenados, dada a grande eficiência da FBN, nessa cultura. No entanto, é importante destacar que os estudos de FBN na soja, desde o início, estiveram associados ao melhoramento genético, $\mathrm{o}$ que não ocorreu com o feijão. É nesse aspecto que o melhoramento poderia ser útil, pois existem evidências científicas de que é possível melhorar o caráter nodulação (Herridge \& Rose 2000, Alcântara et al. 2009). Assim, com a limitação atual de cultivares de feijão eficientes na FBN, é possível utilizar métodos de cultivo que propiciem incremento nesse caráter.

Herridge et al. (2001) citam que o incremento na FBN, por meio do melhoramento genético e do manejo eficiente, traz alto benefício econômico. Tais perspectivas podem viabilizar a cultura, tanto no sentido agronômico como na sustentabilidade agrícola, até ocorrer o lançamento de cultivares mais eficientes na FBN.

\section{CONCLUSÕES}

1. O uso de extrato de alga, molibdênio e pó de rocha, associados à inoculação, propicia maior eficiência nos caracteres emergência e nodulação, quando comparados ao método convencional de cultivo.

2. A inoculação, por si só, não é suficiente para atingir rendimento de grãos em patamares similares aos do sistema convencional. Porém, quando associada ao pó de rocha, molibdênio e extrato de alga, o rendimento de grãos não difere do método convencional. 
3. O método de cultivo com inoculação e complementação via sementes com extrato de alga, molibdênio e pó de rocha proporciona manutenção no rendimento de grãos com menor custo, em relação ao método de cultivo convencional.

\section{REFERÊNCIAS}

ALCÂNTARA, R. M. C. M. et al. Estado atual da arte quanto à seleção e o melhoramento de genótipos para a otimização da FBN. Teresina: Embrapa Meio-Norte, 2009.

ALVES, B. J. R.; BODDEY, R. M.; URQUIAGA, S. The success of BNF in soybean in Brazil. Plant and Soil, Dordrecht, v. 252, n. 1, p. 1-9, 2003.

ASSOCIAÇÃO AÇÃO NASCENTE MAQUINÉ/ UNIVERSIDADE FEDERAL DO RIO GRANDE DO SUL (Anama/UFRGS). Diagnóstico socioeconômico e ambiental do Município de Maquiné - RS: perspectivas para um desenvolvimento rural sustentável. Porto Alegre: UFRGS, 2000.

BARBOSA FILHO, M. P.; FAGERIA, N. K.; ZIMMERMANN, F. J. P. Atributos de fertilidade do solo e produtividade do feijoeiro e da soja influenciados pela calagem em superfície e incorporada. Ciência e Agrotecnologia, Lavras, v. 29, n. 3, p. 507-514, 2005.

BINOTTI, F. F. S. et al. Fontes, doses e modo de aplicação de nitrogênio em feijoeiro no sistema plantio direto. Bragantia, Campinas, v. 68, n. 2, p. 473-481, 2009.

BISCARO, G. A. et al. Nitrogênio em cobertura e molibdênio via foliar no feijoeiro irrigado cultivado em solo de Cerrado. Acta Scientiarum Agronomy, Maringá, v. 33, n. 4, p. 665-670, 2011.

CAMARGO, C. K. et al. Produtividade do morangueiro em função da adubação orgânica e com pó de basalto no plantio. Semina: Ciências Agrárias, Londrina, v. 33, supl. 1, p. 2985-2994, 2012.

COMISSÃO TÉCNICA SUL-BRASILEIRA DE FEIJÃO (CTSBF). Informações técnicas para o cultivo do feijão na Região Sul brasileira 2009. Florianópolis: Epagri, 2010.

COMPANHIA NACIONAL DE ABASTECIMENTO (Conab). Acompanhamento da safra brasileira de grãos. 2014. Disponível em: $<$ http://www.conab.gov.br $>$. Acesso em: 05 set. 2014.

CREWS, T. E.; PEOPLES, M. B. Legume versus fertilizer sources of nitrogen: ecological trade-offs and human needs. Agriculture, Ecosystems \& Environment, Amsterdam, v. 102, n. 3, p. 279-297, 2004.

DURAND, N.; BRIAND, X.; MEYER, C. The effect of marine bioactive substances (NPRO) and exogenous cytokinins on nitrate reductase activity in Arabidopsis thaliana. Physiologia Plantarum, London, v. 119, n. 4, p. 489-493, 2003.

FERREIRA, E. R. N. C.; ALMEIDA, J. A.; MAFRA, A. L. Pó de basalto, desenvolvimento e nutrição do feijão comum (Phaseolus vulgaris L.) e propriedades químicas de um Cambissolo Húmico. Revista de Ciências Agroveterinárias, Lages, v. 8, n. 2, p. 111-121, 2009.

GERLACH, G. A. X. et al. Análise econômica da produção de feijão em função de doses de nitrogênio e coberturas vegetais. Pesquisa Agropecuária Tropical, Goiânia, v. 43, n. 1, p. 42-43, 2013.

GOPALAKRISHNAN, S. et al. Plant growth promoting rhizobia: challenges and opportunities. 3 Biotech, Berlin, v. 5, n. 4, p. 355-377, 2014.

GRANGE, L. et al. New insights into the origins and evolution of rhizobia that nodulate common bean (Phaseolus vulgaris L.) in Brazil. Soil Biology \& Biochemistry, Brisbaine, v. 39, n. 4, p. 867-876, 2007.

HERRIDGE, D. E.; ROSE, I. Breeding for enhanced nitrogen fixation in crop legumes. Field Crops Research, Amsterdam, v. 65, n. 2-3, p. 229-248, 2000.

HERRIDGE, D. F.; TURPIN, J. E.; ROBERTSON, M. J. Improving nitrogen fixation of crop legumes through breeding and agronomic management: analysis with simulation modeling. Australian Journal of Experimental Agriculture, Collingwood, v. 41, n. 3, p. 391-401, 2001.

HUNGRIA, M.; VARGAS, M. A. T. Environmental factors affecting $\mathrm{N}_{2}$ fixation in grain legumes in the tropics with an emphasis on Brazil. Field Crops Research, Amsterdam, v. 65, n. 2-3, p. 151-164, 2000.

INSTITUTO DE ECONOMIA AGRÍCOLA (IEA). Coordenadoria de Assistência Técnica Integral. Planilha de cálculo de custo operacional de trator-implemento. 2014. Disponível em: <http://www.cati.sp.gov.br/new/ acervotecnico.php?ID=31>. Acesso em: 10 out. 2014.

KHAN, W. et al. Seaweed extracts as biostimulants of plant growth and development. Plant Growth Regulation, Dordrecht, v. 28, n. 4, p. 386399, 2009.

LEMOS, L. B.; FERREIRA, D. C.; FARINELLI, R. Desempenho agronômico, nutricional e tecnológico de cultivares de feijoeiro adubados com nitrogênio em cobertura no sistema de plantio direto. In: CONGRESSO NACIONAL DE PESQUISA DE FEIJÃO, 9., 2008, Campinas. Anais... Campinas: IAC, 2008. p. 1400-1403.

LOPES, J. F. et al. Adubação foliar com níquel e molibdênio no feijoeiro comum cv. Ouro Vermelho. Ceres, Viçosa, v. 61, n. 2, p. 234-240, 2014.

MATOSO, S. C. G.; KUSDRA, J. F. Nodulação e crescimento do feijoeiro em resposta à aplicação de 
molibdênio e inoculante rizobiano. Revista Brasileira de Engenharia Agrícola e Ambiental, Campina Grande, v. 18, n. 6, p. 567-573, 2014.

MATYSIAK, K.; KACZMAREK, S.; KRAWCZYK, R. Influence of seaweed extracts and mixture of humic and fulvic acids on germination and growth of Zea mays $\mathrm{L}$. Acta Scientiarum Polonorum, Lublin, v. 10, n. 1, p. 3345, 2011.

MATZENAUER, R.; RADIN, B.; ALMEIDA, I. R. (Eds.). Atlas climático: Rio Grande do Sul. Porto Alegre: Fepagro, 2011.

NEVES, M. C. P. et al. Por que não utilizar ureia como fonte de N na agricultura orgânica. Cadernos de Ciência \& Tecnologia, Brasília, DF, v. 19, n. 2, p. 313-331, 2002.

PELEGRIN, R. et al. Resposta da cultura do feijoeiro à adubação nitrogenada e à inoculação com rizóbio. Revista Brasileira de Ciência do Solo, Viçosa, v. 33, n. 1, p. 219226, 2009.

PLEWKA, R. G. et al. Avaliação do uso do pó de basalto na produção de feijão. Revista Brasileira de Agroecologia, Cruz Alta, v. 4, n. 2, p. 4397-4400, 2009.

ROCHA, P. R. R. et al. Adubação molíbdica na cultura do feijão nos sistemas de plantio direto e convencional. Revista Caatinga, Mossoró, v. 24, n. 1, p. 9-17, 2011.

ROSSI, R. L. et al. Adubação foliar com molibdênio na cultura da soja. Journal of Agronomic Sciences, Umuarama, v. 1, n. 1, p. 12-23, 2012.

SCHOONHOVEN, A. V.; PASTOR-CORRALES, M. A. Standard system for the evaluation of bean germoplasm. Cali: CIAT, 1987.
SANTOS, G. M. et al. Características e rendimento de vagem do feijão-vagem em função de fontes e doses de matéria orgânica. Horticultura Brasileira, Brasília, DF, v. 19, n. 1, p. 30-35, 2001.

SAS INSTITUTE INC. SAS ${ }^{\circledR}$ university edition: installation guide for Windows. Cary: SAS Institue, 2014.

SILVA, A. et al. Fertilidade do solo e desenvolvimento de feijão comum em resposta a adubação com pó de basalto. Revista Brasileira de Ciências Agrárias, Lages, v. 7, n. 4, p. 548-554, 2012.

SOUZA, P. M.; MOREIRA, F. M. S. Potencial econômico da inoculação de rizóbios em feijão-caupi na agricultura familiar: um estudo de caso. Em Extensão, Uberlândia, v. 10, n. 2, p. 37-54, 2011.

THEODORO, S. H.; LEONARDOS, O. H. The use of rocks to improve family agriculture in Brazil. Anais da Academia Brasileira de Ciências, Rio Janeiro, v. 78, n. 4, p. 721-730, 2006.

TOZLU, E. et al. Effect of some plant growth promoting bacteria on yield components of dry bean (Phaseolus vulgaris L. cv. Aras 98). Journal of the Agricultural Faculty, Fukuoka, v. 43, n. 2, p. 101-106, 2012.

VASCONCELOS, B. M. F.; GONÇALVES, A. A. Macroalgas e seus usos: alternativas para as indústrias brasileiras. Revista Verde, Pombal, v. 8, n. 5, p. 125-140, 2013.

WANDER, A. E.; ABREU, A. F. B. Cultivo do feijão da primeira e segunda safras na região sul de Minas Gerais. Santo Antônio de Goiás: Embrapa Arroz e Feijão, 2005. (Sistemas de produção, 6). 